\title{
Efficiency Evaluation of Primary and Secondary Education Sector Performance in East Java Using Data Envelopment Analysis
}

\author{
Bahalwan Apriyansyah $^{1}$, Syarifa Hanoum ${ }^{2}$, Prahardika Prihananto ${ }^{3}$, Siti Lailatul Mukaromah ${ }^{4}$ \\ 1,2,3,4 Faculty of Creative Design and Digital Business, Institut Teknologi Sepuluh Nopember, Surabaya, \\ 60111 \\ 'bahalwan@its.ac.id \\ ${ }^{2}$ syarifa@mb.its.ac.id \\ 3prahardika@its.ac.id \\ 4lailamukaromah17@gmail.com
}

Received: 11/11/2020.

Reviewed: $16 / 12 / 2020$.

Published: 31/12/2020.

Copyright $@ 2020$ by the author (et al) and Jurnal Sosial Humaniora (JSH)

*This work is licensed under the Creative Commons Attribution International License (CC BY 4.0).

http://creativecommons.org/licenses/by/4.0/
Subject Area : Education, Management

\begin{abstract}
Education has an important role in creating excellent human resources for the advancement of national development. However, in Indonesia, there are still gaps in equal access to education. This study aims to evaluate the performance efficiency of the education sector to provide recommendations of which considered inefficient by using the Data Envelopment Analysis (DEA) method. This study measures the technical efficiency of primary and both secondary schools (junior and senior high School) in 38 regencies/cities in East Java Province for three academic years (2016-2019). The results showed that technical inefficiency still occurs in the implementation of education in East Java. In addition, peer groups and improvement targets were obtained for the inefficient regencies/cities. The indicators that most influence the efficiency value of the education sector in East Java are the Education Fund Allocation as the input variable and the School Continuing Rate as the output variable. The scale efficiency analysis shows that most of the education sector in East Java operates in decreasing returns to scale.
\end{abstract}

Keywords: DEA; Efficiency; Regency/City; Elementary School; Junior High School; High School

\section{Introduction}

The progress of a country's development is largely determined by the quality of its human resources in managing it. Education is one of the key in building an excellent human resources. In 2018, Indonesian Human Capital Index in ranked 87 out of 157 countries, this shows that the contribution of Indonesian human resources is still low to the country's development (The World Bank, 2018). In the same year, the results of Programme for International Student Assessment (PISA) also show that Indonesian Students academic abilities are still far below the international average. Indonesia is still in the category of 10 countries with the lowest ranking of PISA (OECD, 2018). One of the efforts by the government to improve the quality of education in Indonesia is by allocating a large amount of funds, that is $20 \%$ of the State Budget to the education sector. However, this policy has yet to see an optimal impact, indicated by the value of the Net Participation Rate (NER) and the increase of the Human Development Index (HDI) which still is not evenly distributed in each province and regency/city in Indonesia (Kementerian Keuangan 
Republik Indonesia, 2019). The Minister of Finance Sri Mulyani said it was due to inaccuracies in the distribution and management of the education budget (Novelino, 2019).

East Java is a province in Indonesia with the largest number of cities/regencies, consisting of 9 cities and 29 regencies. In 2019, the Human Development Index (HDI) of East Java was still below the national HDI average, scored 71.50 with a national average of 71.92 (Badan Pusat Statistik, 2020). In addition, East Java's HDI is also the lowest of the provinces in Java Island. The HDI is an index that measures the ability of the population to access education, income, and healthcare from the results of development. Among the three, education holds the lowest index in East Java. This condition shows that there are still problems in the education sector in East Java.

Table 1. Human Development Index in Java Island (2019).

\begin{tabular}{|l|c|c|c|c|c|}
\hline \multicolumn{1}{|c|}{ Province } & $\begin{array}{c}\text { Life Expectancy } \\
\text { (Year) }\end{array}$ & $\begin{array}{c}\text { School Life } \\
\text { Expectancy } \\
\text { (Year) }\end{array}$ & $\begin{array}{c}\text { Average } \\
\text { Schooling Years } \\
\text { (Year) }\end{array}$ & $\begin{array}{c}\text { Outcome per } \\
\text { Capita (Rp.) }\end{array}$ & HDI \\
\hline DKI Jakarta & 72,79 & 12,97 & 11,06 & 18.527 & 80,76 \\
\hline DI Yogyakarta & 74,92 & 15,58 & 9,38 & 14.394 & 79,99 \\
\hline Banten & 69,84 & 12,88 & 8,74 & 12.267 & 72,44 \\
\hline Jawa Barat & 72,85 & 12,48 & 8,37 & 11.152 & 72,03 \\
\hline Jawa Tengah & 74,23 & 12,68 & 7,53 & 11.102 & 71,73 \\
\hline Jawa Timur & 71,18 & 13,16 & 7,59 & 11.739 & 71,50 \\
\hline
\end{tabular}

Note: “Jawa Timur" stands for East Java

According to Ramliyanto, the secretary of East Java Regional Education Office, the low score of the education index in HDI is due to the high gap in the School Participation Rate and the distribution of teaching staff (Nasafi, 2019). In addition, according to the strategic plan of East Java Regional Education Office for 2014-2019, there are several main problems of education in East Java including: 1) disparity in the quality of education is still high; 2) the quality of teaching staff is still low; 3) the quantity and quality of educational facilities and infrastructure is still low; 4) the quality of non-formal and informal education is still low; 5) the level of literacy and interest in reading is still low; 6) the Gross Enrollment Rate (GER) and Net Enrollment Rate (NER) are still low, especially in secondary education; 7) the lack of infrastructure for vocational secondary education; 8) the lack of teaching staff in special education and special services (Dinas Pendidikan Jawa Timur, 2014).

Figure 1. GER (APK) dan NER (APM) in East Java for 2018/2019

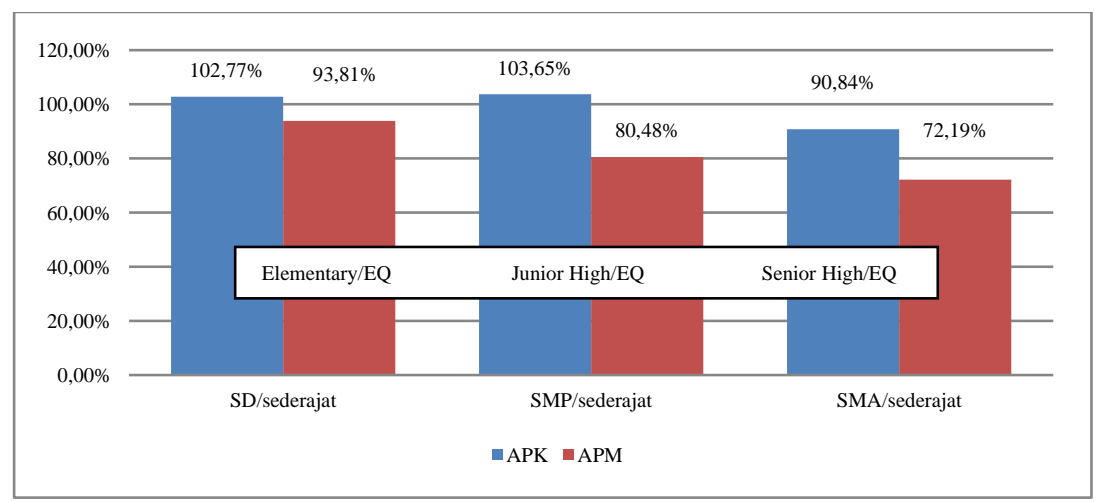


One of the efforts of the East Java Provincial Government in overcoming these problems is by allocating 30 percent of the total Regional Budget (APBD) for the education sector in order to increase equal access to education and reduce dropout rates so as to improve the quality of education in East Java (Soekarno, 2020). Evaluation of performance efficiency in the education sector in Indonesia is important considering the large allocation of education funds. Performance measurement in the education sector aims to understand the process of activity management, ensure objective decision-making, assist the government and those responsible for improving performance and hold them accountable to the public (Mahsun et al., 2011).

This study aims to evaluate the performance efficiency of primary and secondary education sector in each city/regency in East Java so that the allocation of resources used is right on target so as to increase equal access to education. The results of this study are in the form of evaluation, determining indicators of the most influential variables, and recommendations for improving performance efficiency, all from technical cost efficiency, technical system efficiency, and overall efficiency in the education sector in each city/regency in East Java. The results of this study can also be used as a reference for other provinces that have similar problems in the education sector in order to increase the efficiency of their performance.

\section{Literature Review}

\section{Performance}

Performance is the achievement of individual or group work from an organization or entity based on responsibility and authority to achieve legal organizational goals (Sutrisno, 2010). Another definition of performance is the result of correction of a job with the target achievements have already been made (Rai, 2008).

\section{Efficiency Based Performance Measurement}

Efficiency is the ratio of output to input. performance is considered efficient when (1) output can be increased from the use of constant input, (2) output is constant even with less input, and (3) output increases with the increases of input (Suswadi, 2007). Efficiency is a performance parameter that underlies the entire performance of an organization (Hadad et al., 2003).

\section{Data Envelopment Analysis (DEA)}

DEA is a linear programming used in measuring performance using the ratio of output to input from a Decision Making Unit (DMU) or production entity (Suliasih et al., 2013). DMU is a term for a unit or organization that produces an output using certain inputs. A DMU is considered to have the best performance (efficient) if they have a score of 1 or $100 \%$, while considered inefficient if they have a score between 0 and 100\% (Ramanathan, 2003).

There are two DEA models, namely DEA-CCR or CRS (Constant Returns to Scale) and DEA-BCC or VRS (Variable Returns to Scale). This study uses the DEA CRS and DEA VRS models with output orientation. The DEA VRS model assumes that not all DMUs work optimally and there are differences in 
scale between DMUs. The DEA CRS model in this study is used to obtain the scale efficiency (SE) value by comparing the technical efficiency value of the CRS (TECRS) model with the VRS (TEVRS) model.

Figure 2. DEA Scale Efficiency

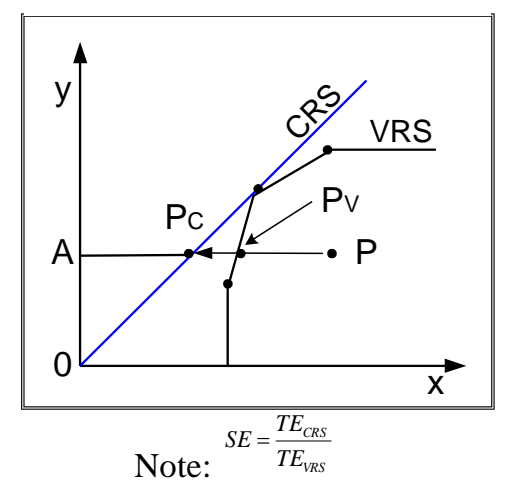

The value of one (efficient DMU) in SE is obtained if TECRS value is the same as TEVRS. If the SE value is more than one, it indicates that the DMU has a scale inefficiency. TEVRS $>$ SE indicates an increase or decrease in efficiency is influenced by pure technical efficiency. Meanwhile, TEVRS $<$ SE shows that the change in efficiency is more influenced by scale efficiency (Worthington, 2000).

\section{Peer Group}

Peer group is the determination of reference DMU for inefficient DMUs in order to achieve better efficiency (Nugroho et al., 2011). DMUs with a relatively low level of efficiency can be improved by referring to the relatively more efficient DMUs. The target of improvement in both input and output is calculated by multiplying the peer group value with the DMU input or output used as the reference.

\section{Efficiency Evaluation in the Education Sector}

Evaluation of efficiency in education generally discusses the attainment of broad access to education with optimal educational facilities. Access to education is the level of convenience for the school age population to get an education. This convenience is in the form of educational services and infrastructure which include the construction of school units, classrooms, libraries, laboratories, and the availability of teachers and educators that are evenly distributed across regions (Tsani et al., 2017).

\section{Methodology}

\section{Research Stages}

There are four stages in this research. First, the preparation stage in the form of problem identification and defining research objectives by conducting literature studies. Second, the model specification stage. Data collection uses secondary data accessed from the East Java Regional Education Office and the Ministry of Education and Culture publications. The methods used are CRS and VRS Data Envelopment Analysis (DEA) with orientation on output to measure technical cost efficiency, technical system efficiency, and overall efficiency. The third stage is the implementation of the model in the form of determining the 
efficient DMU, peer group, target improvement, and the most influential variables. The fourth and the last stage is the analysis and conclusions of the research.

\section{Determining the DMUs}

The DMUs in this study is determined based on the number of cities/regencies in East Java, which consists of 9 cities and 29 regencies.

\section{Determining the Variables}

The determination of input, intermediate, and output variables in this study is based on the vision, mission, goals, and strategic targets of the Ministry of Education and Culture for the years 2015-2019, the 2014-2019 East Java Regional Education Office Strategic Plan, and previous studies which were then adjusted based on the availability of data that can be accessed from the East Java Regional Education Office. Input variable EFA in this study uses a budget for education affairs consisting of regional funds and regional transfer funds for education divided by the number of students with the assumption allocation student is the same.

Intermediate variables are used to relate input variables and output variables. Intermediate variables consist of the Teacher per Student Ratio (TSR), the Class per Student Ratio (CSR), and the Net Enrollment Rate (NER). The commonly used ratios are student/teacher ratios and student/class ratios, but in this study the opposite is true because of the use of the DEA output-oriented model in order to avoid the risk of bias. The values of TSR and CSR are relatively small, henceforth necessary to multiply them by 1000 to accommodate the use of DEA which can only use two decimal digits maximum.

The output variable uses the School Continuing Rate (SCR) and the Non-Dropping Rate (NDR). SCR is the proportion between the number of new grade 1 students at a certain level of education and the number of graduates the lower level of education. The use in the analysis for Elementary and Junior High School levels will be different from Senior High School levels which use the Graduation Rate (GR) indicator because Senior High School is the highest level in secondary education. Dropout Rate (DR) is the percentage of students who are no longer attending school or have not completed their education, consequently NDR indicator uses the 100-DR formula.

Figure 3. Research Model

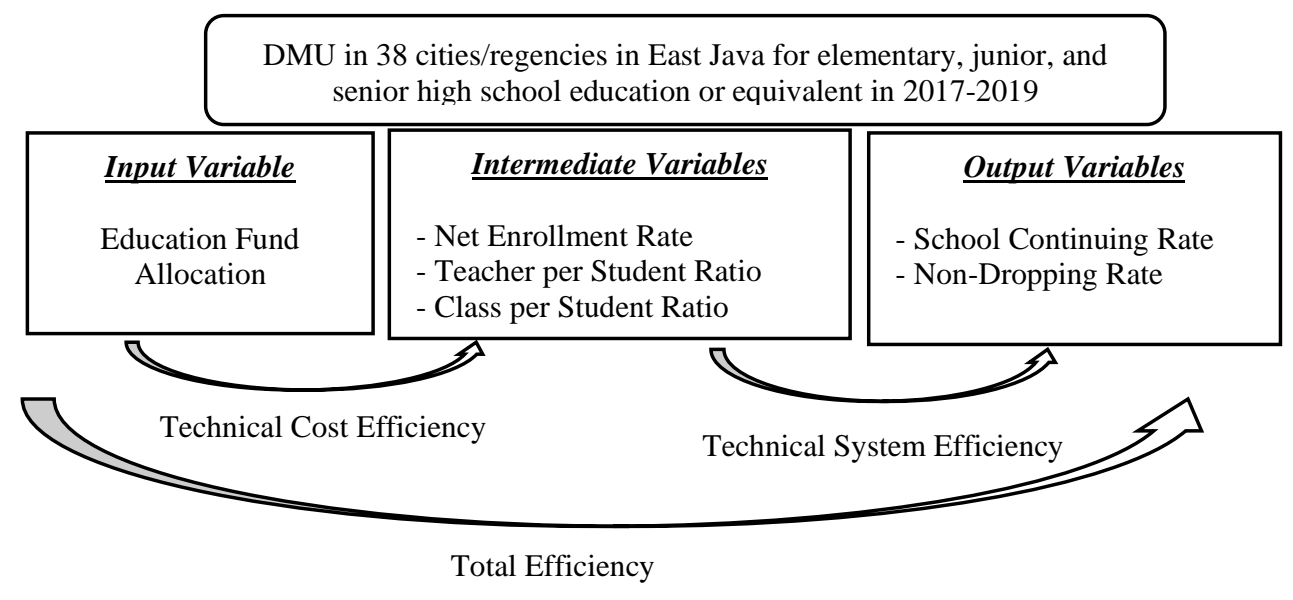




\section{Technical Cost Efficiency (TCE)}

Objective function:

$\operatorname{Max}$

$$
\theta+\varepsilon\left(\sum_{i} I S_{i}+\sum_{j} O S_{j}\right)
$$

Constraining Function:

$\begin{array}{lll}\text { Output } 1 & \text { : Teacher/Student Ratio } & \sum j y_{1 n} \lambda n-\theta_{n} y_{10}-o S_{1}=0 \\ \text { Output } 2 & : \text { Class/Student Ratio } & \sum j y_{2 n} \lambda n-\theta_{n} y_{20}-o s_{2}=0 \\ \text { Output } 3 & \text { : Net Enrollment Rate } & \sum j y_{3 n} \lambda n-\theta_{n} y_{30}-o s_{3}=0 \\ \text { Input } 1 & \text { : Education Fund Allocation } & \sum i X_{1 n} \lambda n+I S_{1}=x_{10}\end{array}$

Index:

$$
\begin{aligned}
n & =\text { DMU, } n=1, \ldots, 38 \\
j & =\text { output }, j=1, \ldots, 3 \\
i & =\text { input }, \quad i=1
\end{aligned}
$$

Data

$y_{j n}=$ Output value to- $j$ from DMU to $n$

$X_{\text {in }}=$ Input value to- $i$ from DMU to $n$

$\varepsilon=$ Small positive number $\left(10^{-6}\right)$

$y j_{0}$ and $x i_{0}$ are the output and input value DMU
Variables

$\theta_{n}=\mathrm{DMU}_{n}$ relative efficiency

$I S_{i} O S_{j}=$ Slack from input I, output $j(\geq 0)$

$\lambda n=$ DMUn value $(\geq 0)$ relative to DMU observed.

\section{Technical System Efficiency}

The calculation of technical system efficiency is conducted by connecting the intermediate variables with the output variables.

Objective Function:

$\operatorname{Max}$

$$
\theta+\varepsilon\left(\sum_{i} I S_{i}+\sum_{j} O S_{j}\right)
$$

Constraining Function:

\begin{tabular}{lll} 
Output 1 & : School Continuing Rate & \multicolumn{1}{c}{$\sum j \mathrm{y}_{1 \mathrm{n}} \lambda n-\theta_{\mathrm{n}} \mathrm{y}_{10}-\mathrm{os}_{1}=0$} \\
Output 2 & $:$ Non-Dropping Rate & $\sum j \mathrm{y}_{2 \mathrm{n}} \lambda n-\theta_{\mathrm{n}} \mathrm{y}_{20}-\mathrm{oS}_{2}=0$ \\
Input 1 & $:$ Teacher/Student Ratio & $\sum i \mathrm{X}_{1 \mathrm{n}} \lambda n+\mathrm{IS}_{1}=\mathrm{x}_{10}$ \\
Input 2 & $:$ Class/Student Ratio & $\sum i \mathrm{X}_{2 \mathrm{n}} \lambda n+\mathrm{IS}_{2}=\mathrm{x}_{20}$ \\
Input 3 & $:$ Net Enrollment Rate & $\sum i \mathrm{X}_{3 \mathrm{n}} \lambda n+\mathrm{IS}_{3}=\mathrm{x}_{30}$
\end{tabular}

\section{Overall Efficiency $(\mathrm{OE})$}

The overall efficiency calculation is conducted by connecting the input, intermediate and output variables. 
Objective Function:

$\operatorname{Max}$

$$
\theta+\varepsilon\left(\sum_{i} I S_{i}+\sum_{j} O S_{j}\right)
$$

Constraining Function:

$\begin{array}{lll}\text { Output } 1 & \text { : School Continuing Rate } & \sum j \mathrm{y}_{1 \mathrm{n}} \lambda n-\theta n y_{1} O-\mathrm{OS}_{1}=0 \\ \text { Output } 2 & : \text { Non-Dropping Rate } & \sum j \mathrm{y}_{2 \mathrm{n}} \lambda n-\theta n y_{2} O-\mathrm{OS}_{2}=0 \\ \text { Input } 1 & : \text { Education Fund Allocation } & \sum i \mathrm{X}_{1 \mathrm{n}} \lambda n+\mathrm{IS}_{1}=\mathrm{x}_{10} \\ \text { Input } 2 & : \text { Teacher/Student Ratio } & \sum i \mathrm{X}_{2 \mathrm{n}} \lambda n+\mathrm{IS}_{2}=\mathrm{x}_{20} \\ \text { Input } 3 & : \text { Class/Student Ratio } & \sum i \mathrm{X}_{3 \mathrm{n}} \lambda n+\mathrm{IS}_{3}=\mathrm{x}_{30} \\ \text { Input } 4 & : \text { Net Enrollment Rate } & \sum i \mathrm{X}_{4 \mathrm{n}} \lambda n+\mathrm{IS}_{4}=\mathrm{x}_{40}\end{array}$

\section{Scale Efficiency}

The difference in the technical efficiency output of $\mathrm{TE}_{\mathrm{CRS}}$ and $\mathrm{TE}_{\mathrm{VRS}}$ indicates the SE value. If the $\mathrm{TE}_{\mathrm{CRS}}$ and $\mathrm{TE}_{\mathrm{VRS}}$ outputs are the same, in other words $\mathrm{SE}=1$, then the DMU is said to be operating optimally.

\section{Results and Discussion}

\section{Efficiency Calculation with DEA-VRS}

Calculations using the DEA-VRS model assume that not all DMUs work optimally, and there are differences in scale between DMUs. DMU is said to be efficient if it has a perfect efficiency value $(\theta=1)$.

Table 2. The values of TCE, TSE, and OE Output Oriented VRS for Elementary School/Equivalents ('kab.' is for 'kabupaten' or regency, and 'kota' is for city)

\begin{tabular}{|c|c|c|c|c|c|c|c|c|c|}
\hline \multirow{2}{*}{ KABUPATEN/KOTA } & \multicolumn{3}{|c|}{ 2016/2017 } & \multicolumn{3}{|c|}{$2017 / 2018$} & \multicolumn{3}{|c|}{$2018 / 2019$} \\
\hline & TCE & TSE & $\mathbf{O E}$ & TCE & TSE & $\mathbf{O E}$ & TCE & TSE & OE \\
\hline Kota Surabaya & 0.9184 & 1.0000 & 1.0000 & 0.9267 & 1.0000 & 1.0000 & 0.9254 & 1.0000 & 1.0000 \\
\hline Kota Malang & 0.9963 & 0.9999 & 1.0000 & 1.0000 & 0.9999 & 1.0000 & 1.0000 & 0.9967 & 1.0000 \\
\hline Kota Madiun & 0.9708 & 1.0000 & 1.0000 & 1.0000 & 1.0000 & 1.0000 & 0.9581 & 1.0000 & 1.0000 \\
\hline Kota Kediri & 0.9598 & 1.0000 & 1.0000 & 0.9635 & 0.9999 & 1.0000 & 0.9498 & 0.9996 & 1.0000 \\
\hline Kota Mojokerto & 0.9620 & 1.0000 & 1.0000 & 1.0000 & 1.0000 & 1.0000 & 1.0000 & 1.0000 & 1.0000 \\
\hline Kota Blitar & 0.9550 & 1.0000 & 1.0000 & 0.9651 & 1.0000 & 1.0000 & 0.9999 & 1.0000 & 1.0000 \\
\hline Kota Pasuruan & 0.9819 & 1.0000 & 1.0000 & 1.0000 & 0.9993 & 1.0000 & 1.0000 & 0.9997 & 1.0000 \\
\hline Kota Probolinggo & 0.9721 & 1.0000 & 1.0000 & 0.9726 & 1.0000 & 1.0000 & 0.8610 & 1.0000 & 1.0000 \\
\hline Kota Batu & 0.9671 & 1.0000 & 1.0000 & 0.9801 & 1.0000 & 1.0000 & 1.0000 & 0.9998 & 0.9998 \\
\hline Kab. Gresik & 0.9924 & 0.9999 & 1.0000 & 0.9907 & 0.9999 & 0.9999 & 0.9950 & 0.9994 & 0.9994 \\
\hline Kab. Sidoarjo & 0.9636 & 1.0000 & 1.0000 & 0.9598 & 1.0000 & 1.0000 & 0.9549 & 0.9994 & 1.0000 \\
\hline Kab. Mojokerto & 1.0000 & 1.0000 & 1.0000 & 1.0000 & 1.0000 & 1.0000 & 0.9973 & 1.0000 & 1.0000 \\
\hline Kab. Jombang & 0.9544 & 1.0000 & 1.0000 & 0.9608 & 1.0000 & 1.0000 & 0.9629 & 0.9997 & 0.9997 \\
\hline Kab. Bojonegoro & 0.9892 & 0.9999 & 0.9999 & 0.9850 & 0.9999 & 0.9999 & 0.9776 & 0.9999 & 0.9999 \\
\hline Kab. Tuban & 1.0000 & 0.9996 & 0.9997 & 0.9964 & 0.9997 & 0.9997 & 1.0000 & 0.9995 & 0.9995 \\
\hline Kab. Lamongan & 1.0000 & 1.0000 & 1.0000 & 1.0000 & 1.0000 & 1.0000 & 1.0000 & 0.9997 & 0.9997 \\
\hline Kab. Madiun & 0.9458 & 1.0000 & 1.0000 & 1.0000 & 1.0000 & 1.0000 & 1.0000 & 0.9996 & 0.9996 \\
\hline Kab. Ngawi & 0.9555 & 1.0000 & 1.0000 & 0.9473 & 1.0000 & 1.0000 & 0.9399 & 0.9995 & 0.9995 \\
\hline Kab. Magetan & 1.0000 & 0.9998 & 0.9999 & 1.0000 & 0.9999 & 0.9999 & 1.0000 & 0.9996 & 0.9996 \\
\hline Kab. Ponorogo & 0.9324 & 0.9999 & 1.0000 & 0.9285 & 1.0000 & 1.0000 & 0.9012 & 0.9998 & 0.9998 \\
\hline Kab. Pacitan & 0.9744 & 0.9999 & 0.9999 & 0.9821 & 0.9999 & 1.0000 & 0.9593 & 0.9999 & 0.9999 \\
\hline Kab. Kediri & 0.9419 & 1.0000 & 1.0000 & 0.9366 & 1.0000 & 1.0000 & 0.9252 & 0.9996 & 0.9996 \\
\hline Kab. Nganjuk & 0.9219 & 1.0000 & 1.0000 & 0.9209 & 1.0000 & 1.0000 & 0.9083 & 0.9997 & 0.9997 \\
\hline Kab. Blitar & 0.9241 & 0.9994 & 0.9995 & 0.9233 & 0.9996 & 0.9996 & 0.9102 & 1.0000 & 1.0000 \\
\hline Kab. Tulungagung & 0.9716 & 0.9997 & 0.9997 & 0.9682 & 0.9998 & 0.9998 & 0.9393 & 0.9998 & 0.9998 \\
\hline Kab. Trenggalek & 0.9802 & 0.9990 & 0.9990 & 0.9732 & 0.9991 & 0.9991 & 0.9587 & 0.9998 & 0.9998 \\
\hline Kab. Malang & 0.9857 & 0.9995 & 0.9995 & 0.9823 & 0.9996 & 0.9996 & 0.9802 & 0.9997 & 0.9997 \\
\hline Kab. Pasuruan & 0.9196 & 1.0000 & 1.0000 & 0.9070 & 1.0000 & 1.0000 & 0.9074 & 0.9987 & 0.9987 \\
\hline
\end{tabular}




\begin{tabular}{|l|c|c|c|c|c|c|c|c|c|}
\hline \multirow{2}{*}{ KABUPATEN/KOTA } & \multicolumn{3}{|c|}{$\mathbf{2 0 1 6 / 2 0 1 7}$} & \multicolumn{3}{|c|}{$\mathbf{2 0 1 7 / 2 0 1 8}$} & \multicolumn{3}{|c|}{$\mathbf{2 0 1 8 / 2 0 1 9}$} \\
\cline { 2 - 10 } & TCE & TSE & OE & TCE & TSE & OE & TCE & TSE & OE \\
\hline Kab. Probolinggo & 0.9629 & 0.9982 & 0.9983 & 0.9527 & 0.9983 & 0.9983 & 0.9442 & 0.9985 & 0.9985 \\
\hline Kab. Lumajang & 1.0000 & 0.9997 & 1.0000 & 0.9814 & 0.9998 & 0.9998 & 0.9756 & 0.9987 & 0.9987 \\
\hline Kab. Bondowoso & 0.9058 & 0.9986 & 0.9987 & 0.8874 & 0.9987 & 0.9988 & 0.8917 & 0.9992 & 0.9992 \\
\hline Kab. Situbondo & 0.9749 & 0.9984 & 0.9984 & 0.9896 & 0.9985 & 0.9986 & 1.0000 & 0.9987 & 0.9987 \\
\hline Kab. Jember & 1.0000 & 0.9987 & 0.9987 & 0.9882 & 0.9988 & 0.9988 & 0.9777 & 0.9986 & 0.9986 \\
\hline Kab. Banyuwangi & 0.9555 & 0.9998 & 0.9999 & 0.9498 & 0.9999 & 0.9999 & 0.9388 & 0.9994 & 0.9994 \\
\hline Kab. Pamekasan & 0.8494 & 1.0000 & 1.0000 & 0.8314 & 1.0000 & 1.0000 & 0.8524 & 1.0000 & 1.0000 \\
\hline Kab. Sampang & 0.9534 & 0.9964 & 0.9965 & 0.9247 & 0.9966 & 0.9966 & 0.9110 & 0.9973 & 0.9973 \\
\hline Kab. Sumenep & 1.0000 & 0.9977 & 0.9978 & 1.0000 & 0.9978 & 0.9978 & 1.0000 & 0.9988 & 0.9988 \\
\hline Kab. Bangkalan & 0.9078 & 1.0000 & 1.0000 & 0.8925 & 1.0000 & 1.0000 & 0.8850 & 0.9940 & 0.9940 \\
\hline
\end{tabular}

Table 3. The values of TCE, TSE, and OE Output Oriented VRS for Junior High/Equivalents

\begin{tabular}{|c|c|c|c|c|c|c|c|c|c|}
\hline \multirow{2}{*}{ KABUPATEN/KOTA } & \multicolumn{3}{|c|}{ 2016/2017 } & \multicolumn{3}{|c|}{$2017 / 2018$} & \multicolumn{3}{|c|}{ 2018/2019 } \\
\hline & TCE & TSE & $\mathbf{O E}$ & TCE & TSE & $\mathbf{O E}$ & TCE & TSE & $\mathbf{O E}$ \\
\hline Kota Surabaya & 0.9555 & 1.0000 & 1.0000 & 0.9584 & 1.0000 & 1.0000 & 0.9496 & 1.0000 & 1.0000 \\
\hline Kota Malang & 0.9550 & 0.9988 & 0.9996 & 0.9444 & 0.9990 & 0.9998 & 0.9469 & 0.9960 & 0.9960 \\
\hline Kota Madiun & 1.0000 & 0.9998 & 0.9999 & 1.0000 & 0.9999 & 1.0000 & 1.0000 & 0.9984 & 0.9984 \\
\hline Kota Kediri & 0.9728 & 0.9986 & 0.9996 & 0.9770 & 0.9989 & 0.9998 & 0.9935 & 1.0000 & 1.0000 \\
\hline Kota Mojokerto & 0.9936 & 0.9999 & 1.0000 & 0.9746 & 0.9999 & 1.0000 & 1.0000 & 0.9985 & 1.0000 \\
\hline Kota Blitar & 1.0000 & 1.0000 & 1.0000 & 1.0000 & 1.0000 & 1.0000 & 1.0000 & 1.0000 & 1.0000 \\
\hline Kota Pasuruan & 0.9828 & 0.9987 & 1.0000 & 1.0000 & 1.0000 & 1.0000 & 1.0000 & 0.9999 & 1.0000 \\
\hline Kota Probolinggo & 1.0000 & 0.9987 & 0.9995 & 0.9826 & 0.9994 & 1.0000 & 0.9592 & 0.9995 & 1.0000 \\
\hline Kota Batu & 0.9645 & 0.9972 & 1.0000 & 0.9715 & 0.9971 & 0.9981 & 1.0000 & 0.9966 & 0.9966 \\
\hline Kab. Gresik & 0.9008 & 1.0000 & 1.0000 & 0.9267 & 0.9999 & 1.0000 & 0.9736 & 0.9987 & 0.9987 \\
\hline Kab. Sidoarjo & 0.9403 & 1.0000 & 1.0000 & 0.9725 & 1.0000 & 1.0000 & 0.9605 & 1.0000 & 1.0000 \\
\hline Kab. Mojokerto & 0.9424 & 0.9975 & 0.9984 & 0.9511 & 0.9976 & 0.9979 & 0.9571 & 0.9960 & 0.9960 \\
\hline Kab. Jombang & 0.9408 & 0.9991 & 0.9991 & 0.9455 & 0.9988 & 0.9989 & 0.9485 & 1.0000 & 1.0000 \\
\hline Kab. Bojonegoro & 0.9095 & 0.9990 & 0.9991 & 0.9115 & 1.0000 & 1.0000 & 0.9378 & 1.0000 & 1.0000 \\
\hline Kab. Tuban & 0.9636 & 0.9981 & 0.9990 & 0.9975 & 0.9981 & 0.9986 & 0.9829 & 0.9966 & 0.9966 \\
\hline Kab. Lamongan & 0.9764 & 1.0000 & 1.0000 & 0.9451 & 1.0000 & 1.0000 & 0.9852 & 0.9981 & 0.9981 \\
\hline Kab. Madiun & 0.9415 & 0.9996 & 1.0000 & 0.9523 & 0.9997 & 0.9999 & 0.9858 & 0.9975 & 0.9975 \\
\hline Kab. Ngawi & 0.8736 & 1.0000 & 1.0000 & 0.9071 & 1.0000 & 1.0000 & 0.9408 & 0.9990 & 0.9991 \\
\hline Kab. Magetan & 0.9624 & 0.9997 & 0.9997 & 0.9362 & 0.9998 & 0.9998 & 0.9885 & 0.9998 & 0.9998 \\
\hline Kab. Ponorogo & 0.9196 & 0.9976 & 0.9976 & 0.9198 & 0.9978 & 0.9978 & 0.9905 & 0.9982 & 0.9982 \\
\hline Kab. Pacitan & 0.9108 & 0.9980 & 0.9982 & 0.8617 & 0.9998 & 0.9999 & 0.9263 & 1.0000 & 1.0000 \\
\hline Kab. Kediri & 0.9189 & 0.9975 & 0.9977 & 0.9314 & 0.9980 & 0.9980 & 0.9227 & 1.0000 & 1.0000 \\
\hline Kab. Nganjuk & 0.9365 & 0.9956 & 0.9959 & 0.9542 & 0.9958 & 0.9960 & 0.9601 & 0.9966 & 0.9966 \\
\hline Kab. Blitar & 0.8886 & 0.9977 & 0.9977 & 0.9160 & 0.9968 & 0.9968 & 0.9485 & 0.9947 & 0.9947 \\
\hline Kab. Tulungagung & 0.9714 & 0.9975 & 0.9976 & 0.9908 & 0.9977 & 0.9980 & 1.0000 & 0.9977 & 0.9977 \\
\hline Kab. Trenggalek & 1.0000 & 0.9963 & 0.9964 & 0.9962 & 0.9964 & 0.9969 & 1.0000 & 0.9956 & 0.9956 \\
\hline Kab. Malang & 0.8970 & 0.9960 & 0.9960 & 0.9193 & 0.9959 & 0.9959 & 0.9545 & 1.0000 & 1.0000 \\
\hline Kab. Pasuruan & 0.8456 & 1.0000 & 1.0000 & 0.8717 & 1.0000 & 1.0000 & 0.9009 & 1.0000 & 1.0000 \\
\hline Kab. Probolinggo & 0.9570 & 0.9940 & 0.9953 & 0.8831 & 0.9949 & 0.9951 & 0.9686 & 0.9894 & 0.9895 \\
\hline Kab. Lumajang & 1.0000 & 0.9953 & 1.0000 & 0.9016 & 0.9961 & 0.9961 & 0.9534 & 0.9923 & 0.9923 \\
\hline Kab. Bondowoso & 0.9464 & 0.9953 & 0.9955 & 0.8921 & 0.9962 & 0.9964 & 0.9235 & 0.9997 & 1.0000 \\
\hline Kab. Situbondo & 1.0000 & 0.9939 & 0.9940 & 0.9599 & 0.9943 & 0.9944 & 0.9928 & 0.9791 & 0.9791 \\
\hline Kab. Jember & 0.8922 & 0.9956 & 0.9956 & 0.9208 & 0.9955 & 0.9955 & 0.9404 & 1.0000 & 1.0000 \\
\hline Kab. Banyuwangi & 0.9088 & 0.9972 & 0.9974 & 0.9252 & 0.9974 & 0.9974 & 0.9289 & 1.0000 & 1.0000 \\
\hline Kab. Pamekasan & 1.0000 & 0.9959 & 0.9969 & 0.9831 & 0.9971 & 0.9971 & 1.0000 & 0.9997 & 1.0000 \\
\hline Kab. Sampang & 0.7938 & 1.0000 & 1.0000 & 0.8001 & 1.0000 & 1.0000 & 0.9088 & 1.0000 & 1.0000 \\
\hline Kab. Sumenep & 1.0000 & 0.9965 & 0.9965 & 1.0000 & 0.9983 & 0.9983 & 1.0000 & 0.9992 & 0.9993 \\
\hline Kab. Bangkalan & 0.8501 & 0.9990 & 0.9995 & 0.8198 & 0.9992 & 0.9993 & 0.9027 & 0.9950 & 0.9955 \\
\hline
\end{tabular}

Table 4. The values of TCE, TSE, dan OE Output Oriented VRS for High School/Equivalents

\begin{tabular}{|c|c|c|c|c|c|c|c|c|c|}
\hline \multirow{2}{*}{ KABUPATEN/KOTA } & \multicolumn{3}{|c|}{$2016 / 2017$} & \multicolumn{3}{|c|}{$2017 / 2018$} & \multicolumn{3}{|c|}{$2018 / 2019$} \\
\hline & TCE & TSE & OE & TCE & TSE & $\mathbf{O E}$ & TCE & TSE & OE \\
\hline Kota Surabaya & 0.7286 & 1.0000 & 1.0000 & 0.7961 & 1.0000 & 1.0000 & 0.8613 & 1.0000 & 1.0000 \\
\hline Kota Malang & 0.9508 & 0.9992 & 0.9994 & 0.9753 & 0.9958 & 0.9988 & 0.9656 & 0.9992 & 1.0000 \\
\hline Kota Madiun & 1.0000 & 0.9975 & 1.0000 & 1.0000 & 0.9944 & 0.9991 & 1.0000 & 0.9975 & 0.9975 \\
\hline Kota Kediri & 0.9770 & 0.9965 & 0.9999 & 0.9836 & 0.9933 & 0.9983 & 1.0000 & 0.9965 & 1.0000 \\
\hline Kota Mojokerto & 1.0000 & 0.9982 & 0.9988 & 1.0000 & 0.9891 & 0.9958 & 1.0000 & 0.9982 & 1.0000 \\
\hline Kota Blitar & 1.0000 & 0.9952 & 1.0000 & 1.0000 & 0.9916 & 1.0000 & 0.8850 & 0.9952 & 0.9961 \\
\hline
\end{tabular}




\begin{tabular}{|c|c|c|c|c|c|c|c|c|c|}
\hline \multirow{2}{*}{ KABUPATEN/KOTA } & \multicolumn{3}{|c|}{$2016 / 2017$} & \multicolumn{3}{|c|}{$2017 / 2018$} & \multicolumn{3}{|c|}{$2018 / 2019$} \\
\hline & TCE & TSE & OE & TCE & TSE & OE & TCE & TSE & OE \\
\hline Kota Pasuruan & 0.9407 & 0.9993 & 1.0000 & 1.0000 & 0.9933 & 1.0000 & 1.0000 & 0.9993 & 1.0000 \\
\hline Kota Probolinggo & 0.9593 & 0.9970 & 0.9984 & 0.9611 & 0.9945 & 1.0000 & 1.0000 & 0.9970 & 1.0000 \\
\hline Kota Batu & 0.8918 & 0.9966 & 1.0000 & 1.0000 & 0.9959 & 1.0000 & 1.0000 & 0.9966 & 1.0000 \\
\hline Kab. Gresik & 0.7661 & 0.9976 & 1.0000 & 0.7889 & 0.9984 & 1.0000 & 0.9096 & 0.9976 & 0.9999 \\
\hline Kab. Sidoarjo & 0.7278 & 1.0000 & 1.0000 & 0.7884 & 1.0000 & 1.0000 & 0.8192 & 1.0000 & 1.0000 \\
\hline Kab. Mojokerto & 0.7899 & 1.0000 & 0.9992 & 0.8218 & 0.9961 & 0.9980 & 0.8992 & 1.0000 & 1.0000 \\
\hline Kab. Jombang & 1.0000 & 1.0000 & 0.9981 & 1.0000 & 0.9947 & 0.9982 & 0.9581 & 1.0000 & 1.0000 \\
\hline Kab. Bojonegoro & 0.8397 & 0.9994 & 0.9974 & 0.7996 & 0.9940 & 0.9967 & 0.8764 & 0.9994 & 0.9995 \\
\hline Kab. Tuban & 0.6204 & 1.0000 & 0.9991 & 0.6526 & 0.9979 & 0.9994 & 0.8116 & 1.0000 & 1.0000 \\
\hline Kab. Lamongan & 0.8110 & 0.9998 & 0.9999 & 0.8713 & 0.9974 & 0.9996 & 0.9933 & 0.9998 & 1.0000 \\
\hline Kab. Madiun & 0.7358 & 0.9979 & 1.0000 & 0.7510 & 0.9976 & 0.9998 & 0.9461 & 0.9979 & 1.0000 \\
\hline Kab. Ngawi & 0.7201 & 1.0000 & 1.0000 & 0.7361 & 0.9979 & 0.9995 & 0.8588 & 1.0000 & 1.0000 \\
\hline Kab. Magetan & 0.7438 & 0.9968 & 0.9997 & 0.7826 & 0.9979 & 0.9998 & 0.8952 & 0.9968 & 0.9976 \\
\hline Kab. Ponorogo & 0.7693 & 0.9997 & 0.9982 & 0.7834 & 0.9959 & 0.9981 & 0.9119 & 0.9997 & 0.9998 \\
\hline Kab. Pacitan & 0.7805 & 1.0000 & 0.9989 & 0.7802 & 0.9959 & 0.9989 & 0.9791 & 1.0000 & 1.0000 \\
\hline Kab. Kediri & 0.6115 & 1.0000 & 0.9979 & 0.6425 & 0.9971 & 0.9980 & 0.8141 & 1.0000 & 1.0000 \\
\hline Kab. Nganjuk & 0.8198 & 1.0000 & 0.9985 & 0.8647 & 0.9958 & 0.9980 & 0.8871 & 1.0000 & 1.0000 \\
\hline Kab. Blitar & 0.6098 & 0.9972 & 1.0000 & 0.6456 & 0.9965 & 0.9984 & 0.8302 & 0.9972 & 1.0000 \\
\hline Kab. Tulungagung & 0.7108 & 0.9995 & 0.9959 & 0.7598 & 0.9937 & 0.9955 & 0.8081 & 0.9995 & 0.9998 \\
\hline Kab. Trenggalek & 0.8720 & 0.9967 & 0.9985 & 0.9225 & 0.9959 & 0.9987 & 0.9542 & 0.9967 & 0.9976 \\
\hline Kab. Malang & 0.5881 & 1.0000 & 1.0000 & 0.6447 & 1.0000 & 1.0000 & 0.9146 & 1.0000 & 1.0000 \\
\hline Kab. Pasuruan & 0.5531 & 1.0000 & 1.0000 & 0.5844 & 1.0000 & 1.0000 & 0.8280 & 1.0000 & 1.0000 \\
\hline Kab. Probolinggo & 0.6542 & 0.9944 & 0.9943 & 0.6769 & 0.9908 & 0.9959 & 0.9417 & 0.9944 & 0.9946 \\
\hline Kab. Lumajang & 1.0000 & 0.9988 & 1.0000 & 0.6439 & 0.9947 & 0.9988 & 0.8879 & 0.9988 & 1.0000 \\
\hline Kab. Bondowoso & 0.6581 & 0.9953 & 0.9943 & 0.6685 & 0.9909 & 0.9945 & 0.9304 & 0.9953 & 0.9959 \\
\hline Kab. Situbondo & 0.7759 & 0.9945 & 0.9938 & 0.8117 & 0.9878 & 0.9914 & 0.9153 & 0.9945 & 0.9955 \\
\hline Kab. Jember & 0.6488 & 1.0000 & 0.9956 & 0.6800 & 0.9946 & 0.9958 & 0.8441 & 1.0000 & 1.0000 \\
\hline Kab. Banyuwangi & 0.7229 & 0.9987 & 0.9981 & 0.7784 & 0.9947 & 0.9965 & 0.8139 & 0.9987 & 0.9987 \\
\hline Kab. Pamekasan & 1.0000 & 0.9977 & 0.9953 & 1.0000 & 0.9902 & 0.9955 & 1.0000 & 0.9977 & 0.9978 \\
\hline Kab. Sampang & 0.5496 & 1.0000 & 1.0000 & 0.4694 & 1.0000 & 1.0000 & 1.0000 & 1.0000 & 1.0000 \\
\hline Kab. Sumenep & 0.7785 & 0.9963 & 0.9948 & 0.8117 & 0.9898 & 0.9975 & 1.0000 & 0.9963 & 0.9964 \\
\hline Kab. Bangkalan & 0.5429 & 1.0000 & 1.0000 & 0.5483 & 0.9976 & 1.0000 & 0.9193 & 1.0000 & 1.0000 \\
\hline
\end{tabular}

\section{Efficiency Calculation with DEA-CRS}

The calculation of efficiency with the DEA-CRS model in this study is only used to analyze the overall efficiency. There are 4 cities/regencies that have perfect overall efficiency $(\theta=1)$ in three consecutive academic years at Elementary, Junior High, and High School or its equivalents.

\section{Scale Efficiency Analysis}

Table 6. Scale Efficiency in Each Level of Education

\begin{tabular}{|l|c|c|c|}
\hline \multirow{2}{*}{\multicolumn{1}{|c|}{ Education Level }} & \multicolumn{3}{c|}{ Scale Efficiency } \\
\cline { 2 - 4 } & DRS & CRS & IRS \\
\hline Elementary/Equivalent & 83 & 31 & 0 \\
\hline Junior High School/Equivalent & 89 & 25 & 0 \\
\hline Senior High School/Equivalent & 91 & 23 & 0 \\
\hline Total & 263 & 79 & 0 \\
\hline Percentage & $76,90 \%$ & $23,10 \%$ & $0,00 \%$ \\
\hline
\end{tabular}

Most education operation in East Java is on a decrease return to scale (DRS), with $76.90 \%$ of the total value. This condition means that the ratio of increase in output is smaller than the ratio of increase in input and it can be said that most of the efficiency values are not only influenced by pure technical efficiency but also by scale efficiency. 


\section{Peer Group Analysis}

Based on the results of peer group for all levels of education, for cities/regencies that are not efficient at technical cost efficiency can refer to Madiun City, Pamekasan Regency, and Trenggalek Regency. Meanwhile, peer group for technical system efficiency refer to Blitar City, Mojokerto Regency, and Pacitan Regency. Overall efficiency can refer to Blitar City, Batu City, and Gresik Regency.

Figure 4. Peer Group for Technical Cost Efficiency

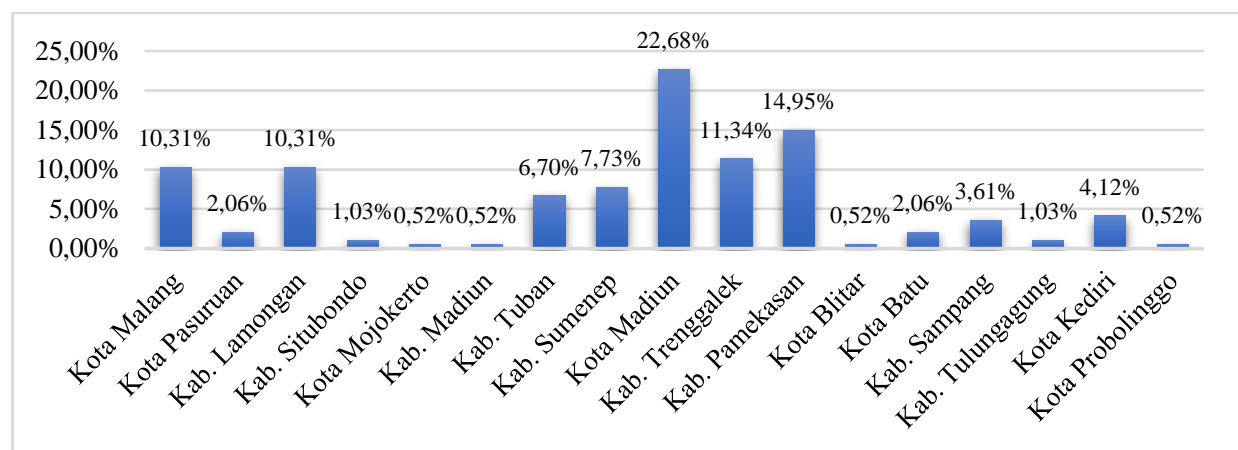

Figure 5. Peer Group for Technical System Efficiency

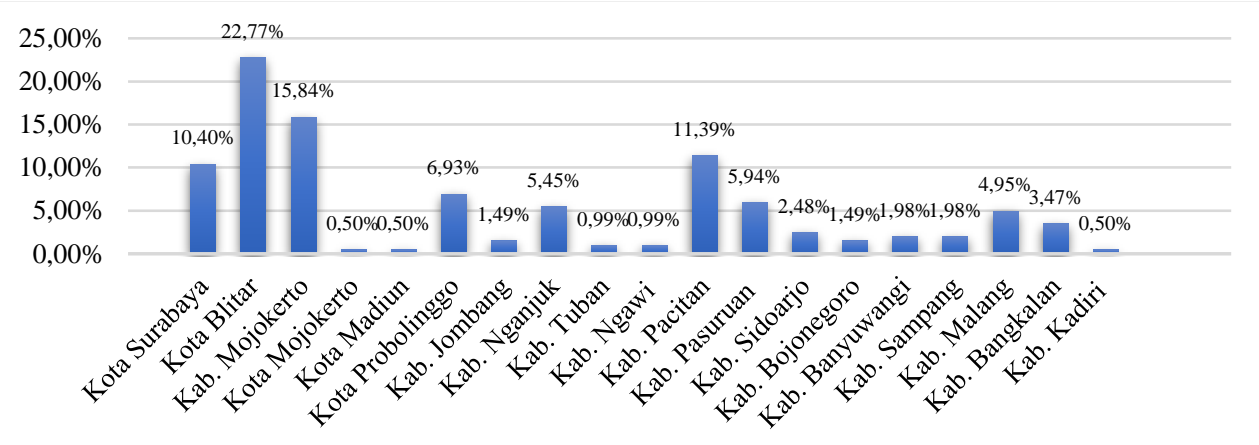

Figure 7. Peer Group for Overall Efficiency

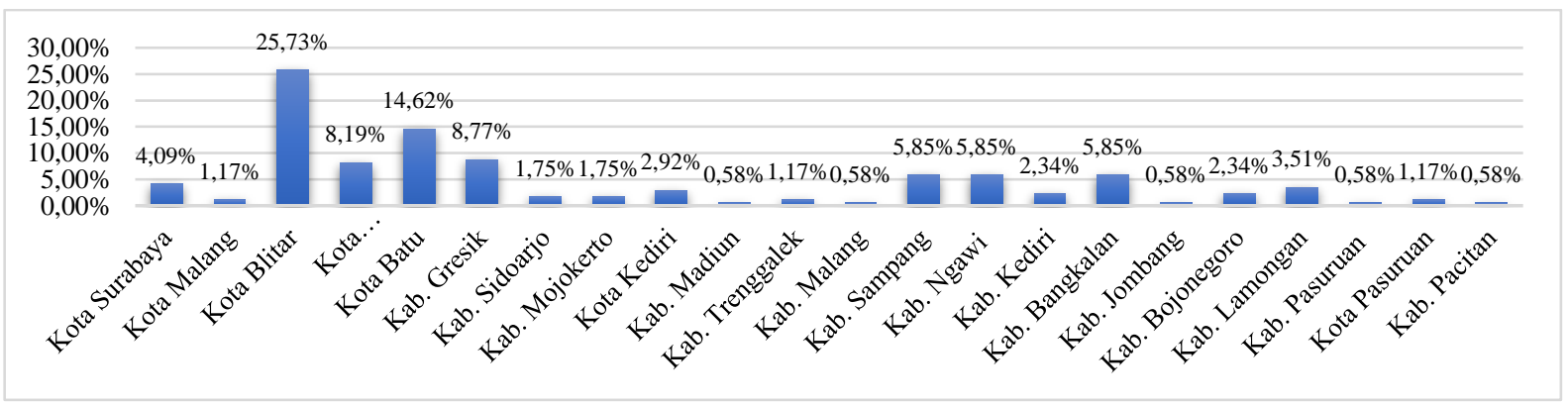

\section{Improvement Targets Analysis}

Inefficient DMUs will get a target of improvement in each of their variables. Even though this research using an output oriented model, the target of improvement could still be a decrease in input due to the slack value for negative input. This study uses two types of targets. 
Strong Projection $(\mathrm{SP})=$ Initial value + proportionate + slack movement

Weak Projection $(\mathrm{WP})=$ Initial value + proportionate

Table 7. Lowest Technical Cost Efficiency Improvement Target at Elementary School Level

\begin{tabular}{|l|l|l|l|l|}
\hline Kab. Pamekasan & \multicolumn{1}{|c|}{ EFA } & \multicolumn{1}{|c|}{ TSR } & CSR & NER \\
\hline Initial Data & Rp355,782 & 93,145 & 52,945 & 81,020 \\
\hline Proportionate & - & 16,133 & 9,170 & 14,033 \\
\hline Slack & - & - & 8,168 & - \\
\hline Weak Projection & Rp355,782 & 109,278 & 62,115 & 95,053 \\
\hline Strong Projection & Rp355,782 & 109,278 & 70,283 & 95,053 \\
\hline
\end{tabular}

\section{Analysis of Variable Influence on Regency/City Efficiency}

The percentage of the influence of variables in determining the efficiency of a DMU is obtained by calculating the average value of each improvement targets and then averaged again. After calculating the average of all variable indicators, the highest average value is the Education Fund Allocation (EFA), which means that EFA has the most influence on the efficiency of education in East Java. Therefore, it is important to re-evaluate the education budget allocation and re-allocate the budget in several districts/cities adjusting to the needs in carrying out education in each district/city in East Java.

Table 8. Variables Most Affecting Efficiency Value of Education Implementation in Each Level

\begin{tabular}{|c|c|c|c|c|c|c|c|c|c|}
\hline \multirow{2}{*}{ Variables } & \multicolumn{4}{|c|}{ Education Level } & \multicolumn{4}{|c|}{ Academic Year } & \multirow{2}{*}{ Rank } \\
\hline & ES & JHS & SHS & Average & 2017 & 2018 & 2019 & Average & \\
\hline EFA & $-6,669 \%$ & $-8,288 \%$ & $-3,166 \%$ & $-6,041 \%$ & $-3,744 \%$ & $-6,345 \%$ & $-8,035 \%$ & $-6,041 \%$ & 1 \\
\hline TSR & $-0,716 \%$ & $0,698 \%$ & $6,098 \%$ & $2,027 \%$ & $3,581 \%$ & $1,483 \%$ & $1,016 \%$ & $2,027 \%$ & 4 \\
\hline CSR & $-0,343 \%$ & $1,389 \%$ & $5,954 \%$ & $2,333 \%$ & $4,925 \%$ & $2,330 \%$ & $-0,255 \%$ & $2,333 \%$ & 3 \\
\hline NER & $1,129 \%$ & $1,769 \%$ & $6,976 \%$ & $3,291 \%$ & $4,314 \%$ & $3,947 \%$ & $1,613 \%$ & $3,291 \%$ & 2 \\
\hline SCR & $0,333 \%$ & $3,628 \%$ & $0,292 \%$ & $1,418 \%$ & $0,647 \%$ & $0,667 \%$ & $2,939 \%$ & $1,418 \%$ & 5 \\
\hline DR & $0,048 \%$ & $0,190 \%$ & $0,217 \%$ & $0,152 \%$ & $0,131 \%$ & $0,175 \%$ & $0,149 \%$ & $0,152 \%$ & 6 \\
\hline
\end{tabular}

\section{Conclusion}

Based on the results of performance efficiency value calculation in the education sector in East Java, the perfect cost technical efficiency value $(\theta=1)$ in 3 consecutive academic years is found in 3 cities/regencies at the levels of Elementary, Junior High, and High School or equivalent. For technical efficiency of the system, there are 7 cities/regencies at the elementary level, and 5 cities/regencies at the junior high and senior high school levels or equivalent. Meanwhile, overall efficiency is found in 11 cities/regencies for elementary school level, 7 cities/regencies for junior high school level, and 8 cities/regencies for senior high school level or equivalent.

Based on the analysis of the influence of variables on the performance efficiency value of the education sector in East Java, it shows that the Education Fund Allocation (EFA) is the most influential indicator. Therefore, it is necessary to calculate in detail the allocation of the education budget that adjusts 
to the needs of education in East Java at each level so that the allocation of these funds can be right on target. Based on the peer group results for districts / cities that are not yet efficient on technical cost efficiency can refer to Madiun City, while for technical system efficiency and overall efficiency it can refer to Blitar City.

\section{References}

Badan Pusat Statistik. (2020). Indeks Pembangunan Manusia (IPM) Tahun 2019 (Berita Resmi Statistik). Badan Pusat Statistik. https://www.bps.go.id/pressrelease/2020/02/17/1670/indeks-pembangunanmanusia--ipm--indonesia-pada-tahun-2019-mencapai-71-92.html

Dinas Pendidikan Jawa Timur. (2014). Rencana Strategis Dinas Pendidikan Jawa Timur Tahun 2014-2019. Dinas Pendidikan Jawa Timur.

Hadad, M. D., Santoso, W., Mardanugraha, E., \& Ilyas, D. (2003). Pendekatan Parametrik Untuk Efisiensi Perbankan Indonesia (Working Paper Bank Indonesia). Bank Indonesia.

Kementerian Keuangan Republik Indonesia. (2019, November 5). Anggaran Pendidikan ke Daerah Direview Agar Berdampak Bagi Peningkatan Kualitas Pendidikan. Kementerian Keuangan. https://www.kemenkeu.go.id/publikasi/berita/anggaran-pendidikan-ke-daerah-direview-agarberdampak-bagi-peningkatan-kualitas-pendidikan/

Mahsun, M., Sulistyowati, F., \& Purwanugraha, H. A. (2011). Akuntansi Sektor Publik (3rd ed.). BPFE.

Nasafi. (2019, July 6). Inilah Isu-Isu Strategis Pembangunan Pendidikan di Jawa Timur. Pwmu.co | Portal Berkemajuan. https://pwmu.co/101347/07/06/inilah-isu-isu-strategis-pembangunan-pendidikan-dijawa-timur/

Novelino, A. (2019, March 12). Sri Mulyani “Kecewa” dengan Pengelolaan Anggaran Pendidikan. CNN Indonesia. https://www.cnnindonesia.com/ekonomi/20190312142919-532-376533/sri-mulyanikecewa-dengan-pengelolaan-anggaran-pendidikan

Nugroho, S., Sriyanto, S., \& Chasanah, N. (2011). ANALISIS EFISIENSI DISTRIBUSI LISTRIK UNIT PELAYANAN JARINGAN DENGAN METODE DATA ENVELOPMENT ANALYSIS (DEA) Studi Kasus di Area Pelayanan Jaringan Kudus, PT. PLN (Persero). J@ti Undip : Jurnal Teknik Industri, 6(1), 47-56. https://doi.org/10.12777/jati.6.1.47-56

OECD. (2018). PISA 2018 Results (Programme for International Student Assessment). Organisation for Economic Co-operation and Development. https://www.oecd.org/pisa/publications/pisa-2018results.htm

Rai, A. G. (2008). Audit Kinerja Pada Sektor Publik: Konsep Praktik Studi Kasus. Salemba Empat.

Ramanathan, R. (2003). An Introduction to Data Envelopment Analysis. SAGE Publications.

Soekarno, R. (2020, January 17). Gubernur Khofifah: 30 Persen dari APBD Jatim Tahun 2020 untuk Pendidikan. Beritajatim.Com. https://beritajatim.com/politik-pemerintahan/gubernur-khofifah-30persen-dari-apbd-jatim-tahun-2020-untuk-pendidikan/

Suliasih, W., Kuswanto, H., \& Susilaningrum, D. (2013). Penerapan Data Envelopment Analysis untuk Efisiensi Kinerja Karyawan pada PT. X [Paper \& Presentation].

Suswadi. (2007). Analisis Efisiensi Perbankan Syariah di Indonesia (Metode Stochastic Frontier Approach) [Undergrad Thesis]. Universitas Islam Indonesia. 
Sutrisno. (2010). Manajemen Sumber Daya Manusia. Kencana.

The World Bank. (2018). Human Capital Index Data 2018 (The Human Capital Project). The World Bank. https://datacatalog.worldbank.org/dataset/human-capital-index

Tsani, T., Ermas, E., \& Febriantoro, A. R. (2017). Efisiensi Belanja Pendidikan Sekolah Menengah Kejuruan dan Pengaruhnya Terhadap Pemenuhan Akses Pendidikan Menengah di Indonesia. Jurnal Anggaran dan Keuangan Negara Indonesia, 2(1), 89-111. https://doi.org/10.33827/

Worthington, A. (2000). Technical Efficiency and Technological Change in Australian Building Societies [Paper]. https://core.ac.uk/download/pdf/10874489.pdf 